\title{
Linfografía mediante imagen por resonancia magnética en modelo animal canino
}

\section{Lymphography by MRI for animal model canine}

\author{
Xavier Leonardo Jaramillo-Chaustre \\ Ph.D. Medicina y Cirugía Animal \\ Universidad de Pamplona \\ Pamplona, Colombia \\ xavier.jaramillo@unipamplona.edu.co
}

\author{
John Jairo Bustamante-Cano \\ Ph.D. Medicina y Cirugía Animal \\ Universidad de Pamplona \\ Pamplona, Colombia \\ jhonjabu@unipamplona.edu.co
}

\author{
Fernando Gómez-Parra \\ M.Sc. Gerencia de Empresas Agrícolas \\ Universidad de Pamplona \\ Pamplona, Colombia \\ fernando.gomez@unipamplona
}

\begin{abstract}
Resumen- Para este estudio se contó con dos grupos, uno de 12 animales sanos y otro con 10 animales que cursaban procesos patológicos en la glándula mamaria. Con los animales anestesiados se emplearon imágenes por resonancia magnética para visualizar los conductos linfáticos y linfonodos de la zona inguinal en series potenciadas en 3D TOF en plano dorsal. En los dos grupos se administró gadopentetato de dimeglumina. Para validar la técnica empleada se consideraron en las imágenes tres estructuras (aire, grasa y músculo) cuya IS mostró diferencia significativa entre tejido glandular mamario (IS 184,8 $\pm \mathbf{2 8 , 2}$ ) y linfonodos regionales (IS de $51,3 \pm 5,4$ ), permitiendo determinar el patrón de referencia para el estudio. Se concluye que el empleo de un equipo de IRM de bajo campo proporciona suficiente información para diferenciar intensidades de señal de linfonodos que recogen linfa de procesos patológicos respecto a linfonodos sanos en caninos.
\end{abstract}

Palabras clave- Canino, conducto linfático, linfonodos, imagen por resonancia magnética, intensidad de señal.

Abstract- For this study were included two groups, one of $\mathbf{1 2}$ healthy animals and another of $\mathbf{1 0}$ animals affected by disease conditions in the mammary gland. With the anesthetized animal were used magnetic resonance imaging to visualize the lymph ducts and lymph nodes in the inguinale area in potentiated series in 3D TOF in dorsal plane. In both groups it was administered gadopentetate dimeglumine. To validate the technique used, were considered in the images three structures (air, fat and muscle) which IS showed significant difference between glandular breast tissue (IS $184.8 \pm 28.2$ ) and regional lymph nodes (IS $51.3 \pm 5,4$ ), allowing to determine the benchmark for the study. It is concluded that the use of a low field equipment of MRI provides enough information to differentiate signal intensities of lymph nodes that collect lymph from pathological processes regarding healthy lymph nodes in dogs

Keywords- Canine, lymphatic duct, lymph node, image by magnetic resonance, signal intensity.

\section{INTRODUCCIÓN}

El sistema linfático funciona como apéndice del cardiovascular, recolectando y transportando las proteínas plasmáticas desde el filtrado capilar hacia la sangre. En la mayor parte de los tejidos y órganos, con algunas excepciones como pueden ser el sistema nervioso central, el cartílago, la médula ósea, el tejido óseo o el timo, los capilares sanguíneos están acompañados por un plexo de capilares linfáticos [1]. En esta estructura anatómica se ha demostrado, además, que desempeña un papel muy relevante en la difusión tumoral [2].

El sistema vascular linfático, en esencia, es un sistema de drenaje, que inicia en los capilares linfáticos, vasos de pequeño calibre con un extremo final ciego, que conducen la linfa (líquido claro resultado del filtrado tisular) desde los espacios extracelulares periféricos hasta los vasos linfáticos cada vez mayores que finalmente convergen en grandes conductos linfáticos (conducto torácico y conducto linfático derecho), que desembocan en las grandes venas de la base del cuello. A lo largo del trayecto de los vasos linfáticos se sitúan unos cúmulos encapsulados de tejido linfoide denominados: linfonodos. La linfa penetra en ellos para ser filtrada a través de los vasos linfáticos aferentes y se reparte en su interior mediante un sistema laberíntico de diminutos canales, denominados senos, los cuales están revestidos por células reticulares aplanadas dispuestas a modo de endotelio, próximas a las cuales se sitúan células fagocitarias. La linfa abandona el nódulo a través de los vasos linfáticos eferentes, quedando 
limpia de material extraño a medida que se filtra por los nódulos linfáticos [3].

En los últimos años, la teoría que explica el drenaje del sistema linfático de la glándula mamaria de la perra ha sido notablemente revisada, concluyendo finalmente que las mamas torácicas 1 y 2 drenan en el linfonodo axilar homolateral y, excepcionalmente, algunos conductos eferentes pueden drenar al linfonodo esternal [4], [5], [6], que llevaría al conducto torácico y, finalmente, a la circulación sanguínea [4], [7]. Por otro lado, las glándulas mamarias abdominales y la glándula mamaria inguinal drenan al linfonodo inguinal homolateral [4], [5], [6], que a su vez va a drenar a los linfonodos ilíacos mediales, luego al tronco común y, finalmente, a la cisterna quilosa [4], [7].

Linfografía por resonancia magnética: la linfoescintilografía ha sido sustituida progresivamente por la linfografía por resonancia magnética (LRM) para la valoración del linfoedema, el estudio de los conductos linfáticos con hipoplasia congénita, la obstrucción maligna, la inflamación o la lesión de los mismos. En este contexto, las IRM pueden ser especialmente útiles en el seguimiento de la microcirugía linfática [8]. Las características morfológicas de los linfonodos normales incluyen la presencia de un hilio graso y contornos regulares de señal o densidad homogénea en IRM y TAC; no obstante, en cáncer de mama con micrometástasis a nódulos linfáticos son comúnmente reportados como linfonodos de tamaño norma [9], [10]. Una mejor resolución en la imagen en cortes transversales, aportada por esta técnica, puede identificar alteraciones morfológicas y cambios en la corteza de cualquiera de los linfonodos que, en un paciente con cáncer de mama, podrían permitir la distinción de la migración metastásica en un linfonodo [11].

El agente de contraste y su aplicación: el Gadopentetato de dimeglumina (GPDM) es un agente de contraste del mismo tipo del gadoterate de meglumina, por lo que ambos pueden emplearse para el cartografiado (mapeo) del linfonodo centinela en una linfografía por resonancia magnética intersticial, así como para la visualización del drenaje linfático desde el punto de inyección [12]. Sin embargo, hay que tener en cuenta que se desconoce la seguridad de la mayoría de los metabolitos de estos agentes de contraste linfotrópicos [12]-[14].
La inyección del agente de contraste puede efectuarse intratumoral, peritumoral, intradérmica o subdérmica, suprayacente al tumor o subareolar [15]. Quienes proponen la inyección intratumoral o peritumoral se basan en el hecho de que permite identificar con mayor precisión los sitios reales de drenaje extra axilar; sin embargo, no sirve para identificar los conductos aferentes, hasta en el $40 \%$ de los casos, el porcentaje de Linfonodo Centinela (LC) localizados es menor y disminuye aún más en pacientes con antecedentes de biopsia en el sitio a inyectar, debido a la fibrosis secundaria y a la falta de difusión del agente de contraste [16]-[17].Quienes prefieren la vía intradérmica y subareolar se basan en el hecho de que tanto el parénquima mamario como la piel de la glándula tienen su origen embriológico en el ectodermo, y que el drenaje linfático mamario de ambos estratos confluye en el plexo subdérmicosubareolar de Sappey y drena a un mismo LC en la axila [18], [16].

Otros autores sostienen que una inyección intradérmica facilita la migración del agente de contraste dentro de las vías linfáticas por efecto de las altas presiones y la densidad del tejido conectivo [12], [19], lo que sumado a un masaje en el sitio de inyección, aumenta la migración del agente de contraste [13].

La inyección subcutánea del agente de contraste en el área periareolar de la glándula mamaria es una de las técnicas más adecuadas para identificar el drenaje en estadios tempranos de tumores mamarios, porque tanto el drenaje linfático de la glándula mamaria como el de la parte superficial de la piel de la zona y el tumor comparten las mismas vías de drenaje hacia el linfonodo axilar[20], [21]-[24]; esto se demuestra al volverse azul y radioactivo el linfonodo centinela, cuando se inyecta el coloide dentro del tumor y el colorante por vía subdérmica [25],[24]. Además, la inyección subdérmica presenta otras ventajas, como el hecho de ser más exacta en la detección de tumores no palpables o múltiples tumores.

Aunque se desconoce el mecanismo exacto de absorción y transporte en el sistema linfático del gadopentetato de dimeglumina, este agente probablemente drena desde el espacio intersticial a las vías linfáticas a través de microvasos linfáticos de paredes delgadas y fenestradas, gracias a la combinación de los efectos de la presión, la os- 
mosis y el volumen, similares a otras sustancias hidrosolubles y de bajo peso molecular [12]-[14,] [24], [26]-[31]. La visualización de cada linfonodo puede mostrar un bajo tránsito y el secuestro de este agente de contraste en las sinusoides de cada linfonodo [13]; La visualización desde el lugar de inyección del agente de contraste hasta el primer relevo ganglionar es de gran importancia en el cartografiado del conducto linfático y del linfonodo al que drena [32]-[34].

\section{MATERIALES Y MÉTODOS}

Para la realización del presente estudio se utilizó un total de 22 individuos de la especie canina, todos ellos hembras, un grupo de 12 animales sanos y otro de 10 animales que cursaban procesos patológicos en glándula mamaria. Con edades comprendidas entre 1 y 11 años y un peso comprendido entre 5,8 y $65,0 \mathrm{~kg}$. Todas ellas habían sido remitidas a la Unidad de Cirugía y Diagnóstico por Imagen (UCDI) de la Facultad de Veterinaria de la Universidad de León (FVULE) por otros centros veterinarios, o bien admitidas directamente en nuestro servicio, por motivos absolutamente ajenos a problemas mamarios, reproductivos 0 endocrinos, esto para los animales sanos; por otra parte los animales con afección de la glándula mamaria fueron los que llegaban a la Unidad de Cirugía para realizarles excéresis de tumor mamario. A los dos grupos se les realizó una exploración exhaustiva de las mamas, incluyendo el estudio imaginológico mediante resonancia magnética, siempre previo consentimiento expreso de sus propietarios.

\subsection{Material fungible empleado en la anestesia}

En el protocolo anestésico utilizado para la realización de los estudios mediante IRM (Imagen por Resonancia Magnética) se administraron soluciones de Midazolam y Buprenorfina, como preanestésicos. Propofol en solución como agente inductor de la anestesia, elegido por su efecto ultracorto y su rápida metabolización y eliminación intra y extrahepática, e isofluorano para el mantenimiento de la anestesia general, así como cal sodada para la absorción de $\mathrm{CO}_{2}$ durante este periodo.

Para la aplicación, fijación y suministro de fármacos anestésicos y fluidos se emplearon caté- teres de teflón de calibre 24, 22 y 20 g, además de compresores de caucho para facilitar la visualización de la vena, jeringuillas de distintos tamaños, agujas de diferentes diámetros y longitudes, sistemas de suero y suero salino fisiológico, así como sondas endotraqueales con manguito de neumotaponamiento de diferentes calibres para adaptarse a los diámetros traqueales correspondientes a perros de 6 a $65 \mathrm{Kg}$ de peso, siguiendo la técnica rutinaria descrita en la bibliografía [35], [36], [37]. Toda la información se refleja en la ficha anestésica.

Para la anestesia general de los animales durante la exploración mediante IRM, se utilizó un equipo de anestesia inhalatoria marca MDS MATRIX® (modelo VML), especialmente diseñado para su uso en instalaciones de RM, (no es posible la utilización de un equipo de anestesia convencional, debido a que sus componentes ferromagnéticos se verían afectados por la presencia del imán permanente) con un vaporizador para isofluorano de la marca DRAGER ${ }^{\circledR}$. El equipo se conecta directamente al circuito general de gases del Servicio de Cirugía de la FVULE.

\section{2 Exploración mediante IRM}

Una vez anestesiados, los pacientes se colocaron en la camilla móvil del equipo de RM, con ayuda de diversos posicionadores específicos para el modelo y equipo de RM de que disponíamos, en decúbito supino, con la cabeza en dirección distal con respecto al imán, las extremidades torácicas extendidas en dirección craneal, las pelvianas en dirección caudal y la región por estudiar lo más centrada posible dentro de este. A continuación, se eligió, en función de la talla del paciente, una antena de radiofrecuencia que fue colocada alrededor de la zona que comprende la glándula mamaria inguinal, de tal forma que en las imágenes obtenidas se pudieran visualizar tanto la mama como los conductos linfáticos que drenan la linfa hacia los linfonodos inguinales. Las antenas utilizadas fueron: la de "cuerpo" para los animales de 55 a $65 \mathrm{~kg}$, la de cabeza para los animales de talla media, de 21 a 31kg, y la del tipo 6 pulgadas para los de peso inferior a $20 \mathrm{~kg}$, como describen Gili [38], Millán [35], Godoy [36], Duarte [37] y Jaramillo et al. [39].

Para la realización de los exámenes a través del IRM se emplearon las instalaciones específi- 
cas aisladas mediante cobre en la UCDI, en cuyo interior se encuentra el equipo de RM (GENERAL ELECTRIC MEDICAL SYSTEMS $®)$ modelo SIGNA PROFILE 2, con un imán permanente de 0,2 Teslas.

Para el estudio de los conductos linfáticos y linfonodos inguinales en los pacientes durante el protocolo de exploración, la primera serie que se programó fue el "localizador multiplanar" o de tres planos, serie potenciada en T1 que se obtiene en el plano sagital y cuya función no es otra que encontrar la región anatómica del animal que se va a explorar en el interior del campo magnético. Para la adquisición de estas imágenes se utilizó un TR de $51 \mathrm{~ms}$, un TE de $18 \mathrm{~ms}$, con un espesor de corte de $6 \mathrm{~mm}$ y una separación entre ellos de 2 $\mathrm{mm}$; se utilizó una matriz de adquisición de 256 por 192, que fue empleada en todos los casos del estudio.

Posteriormente, basándonos en el localizador multiplanar, se programaron las distintas series orientándolas manualmente sobre este, prestando especial atención al hecho de que los cortes programados fueran numéricamente suficientes como para cubrir toda la región de interés, así como para que el sentido del corte fuera lo más correcto posible; en nuestro caso, el ángulo empleado fue de $70^{\circ}$, mostrándonos con especial detalle las estructuras de interés tal como describen Jaramillo et al. [39].

Tras obtener el localizador se programó una serie potenciada en T1, para aportar una mejor calidad de imagen anatómica de la región por estudiar, tanto de los conductos linfáticos como de otras estructuras de la región. Con esta serie recopilamos información para su posterior estudio y análisis; en nuestro caso, se emplearon valores de TE que oscilaron entre 32 y 34 ms, mientras que los valores de TR variaron entre 520 y 540 $\mathrm{ms}$, el espacio entre cortes entre 4,5 y $5,5 \mathrm{~mm}$ y el grosor de los cortes entre 5 y $7 \mathrm{~mm}$ [39].

La siguiente serie empleada en nuestro estudio fue la denominada "3D vascular TOF NO MD". Los valores utilizados en cuanto al TE variaron entre 6,6 y 18,6 ms, mientras que el TR fue de $51 \mathrm{~ms}$, con un espesor de corte de $2 \mathrm{~mm}$ y un espaciamiento entre los cortes de $2 \mathrm{~mm}$; el ángulo empleado para esta serie fue de $35^{\circ}$.
Después de esta serie se llevó a cabo una nueva serie 3D TOF con los mismos valores, previa administración del agente de contraste (gadolinio), lo que permite una comparación de las dos series potenciadas en T1 y 3D TOF y 3D TOF posterior al contraste. Los valores empleados para esta serie fueron un TR de $51 \mathrm{~ms}$, un TE de entre 6,6 ms y $18,6 \mathrm{~ms}$, un espesor de corte de $2 \mathrm{~mm}$ y un espaciamiento entre ellos de 2 milímetros.

\subsection{Metodología y validación de la técnica empleada}

Para la validación de la técnica y la metodología empleadas, antes de iniciar las valoraciones efectuamos una serie de mediciones en tres estructuras diferentes, para observar la variación en la escala de grises en una misma estructura y tomarla como referencia posteriormente, además de familiarizarnos de este modo con el programa informático empleado.

Las mediciones fueron realizadas en tres estructuras diferentes: aire, grasa y músculo. En todas las imágenes de nuestro estudio, se trazó un área constante para todas ellas, de $12 \mathrm{~mm}^{2}$, en cada una de las tres estructuras referidas, todas mediante planimetría manual, evaluando de forma automática la medida de la región de interés -ROI- en regiones que se correspondían con aire $(A)$, grasa $(G)$ o músculo $(M)$; como se aprecia en la Fig. 1. y se valoró su intensidad de señal, de acuerdo con la escala de gris para cada región, empleando el programa OSIRIS; en resumen, en cada estructura se efectuó la medición en tres ocasiones y se obtuvo su intensidad de señal de forma automática; se calculó el promedio de las tres medidas realizadas y la media resultante se tomó como valor de referencia para dicha estructura [39].

Para las mediciones de las áreas empleamos el programa informático OSIRIS (versión 4.19), programa especialmente diseñado para la manipulación y análisis de imágenes médicas, desarrollado en el Hospital Cantonal Universitario de Ginebra. Dicho programa fue instalado en un ordenador personal con un procesador Intel ${ }^{\circledR}$ Centrino Duo ${ }^{\circledR}$ de $1,6 \mathrm{GHz}, 2,00 \mathrm{~Gb}$ de memoria RAM y placa gráfica ATI Mobility Radeon $\times 1300$, de 128 $\mathrm{Mb}$ de memoria [39]. 
Las mediciones se realizaron mediante planimetría manual (con ratón óptico con resolución de movimiento de $800 \mathrm{dpi}$ ), usando las herramientas ofrecidas por el programa informático y obteniéndose los resultados de forma automática en lo que se refiere a las medidas de áreas y a los valores de intensidad de grises [39].

Fig. 1. VISUALIZACIÓN DEL TRAZO DE LA ROI (12 $\left.\mathrm{mm}^{2}\right)$ EN LAS DIFERENTES ESTRUCTURAS: AIRE (A), GRASA (G) Y MÚSCULO (M), EN UN CORTE CORONAL

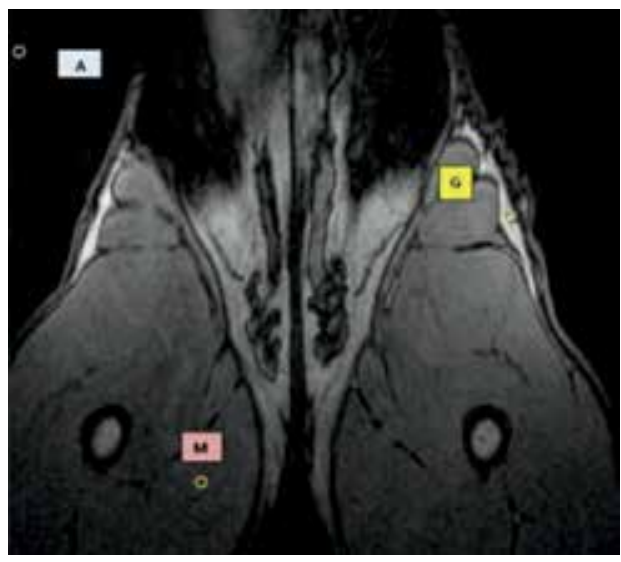

Fuente: Jaramillo y col, 2011.

2.4 Metodología y técnica empleada para establecer las características de intensidad de señal en los linfonodos inguinales en los grupos de estudio (animales sanos y animales procesos patológicos en la glándula mamaria

Dentro de este apartado se estudiaron los linfonodos inguinales en las IRM tanto de las hembras sanas como de las que presentaban afección patológica de las glándulas mamarias; para ello utilizamos la serie 3D TOF, que nos brinda un gran detalle anatómico, tanto del conducto como de los linfonodos. En este caso se utilizó la ROI fija de 12 $\mathrm{mm}^{2}$, debido al reducido tamaño de esta estructura. Dicha medida se aplicó a todas las imágenes de los animales que componen los dos grupos de estudio, antes de inyectar el agente de contraste y después de la inyección del mismo; de esta forma, con una medida fija, obtuvimos un patrón estable para calcular un promedio de estos valores de intensidad de señal y tomarlo como referencia de la estructura en cuestión.

Para la administración del agente de contraste se utilizó el siguiente protocolo: en las glándulas mamarias inguinales derechas se aplicaron 0,5 $\mathrm{ml}$ de gadopentetato de dimeglumina, vía intradérmica en la región sub-areolar de la mama; en las glándulas mamarias inguinales izquierdas se inyectaron $0,5 \mathrm{ml}$ de gadopentetato de dimeglumina más $0,5 \mathrm{ml}$ de $\mathrm{NaCL}$ al $0,9 \%$ en una misma jeringa, con idéntico modo de administración que en las mamas derechas.

El trazo de la ROI fue hecho, tanto en las imágenes precontraste como en las imágenes poscontraste, con lo que se puede establecer la ganancia de contraste en la estructura estudiada.

\subsection{Metodología y técnica empleada para la visualización de los conductos linfáticos que recogen la linfa procedente de las glándulas mamarias abdominal caudal e inguinal en los grupos de estudio}

Para la valoración de los conductos linfáticos que drenan la linfa de las glándulas mamarias abdominal caudal e inguinal en hembras sanas de nuestro estudio nos centramos en las características cualitativas del trayecto de dichos conductos linfáticos, empleando series 3D TOF, por la calidad de las imágenes que aportan en cuanto a visualización de flujos y, en nuestro caso en particular, del conducto linfático.

Las imágenes fueron tomadas en el plano dorsal, que ofrece una mejor visualización del trayecto del conducto, empleando dos protocolos de inyección del agente de contraste: el primero consistió en la administración, vía intradérmica, de la solución de gadopentetato de dimeglumina sin diluir $(0,5 \mathrm{mmol} / \mathrm{ml})$, a dosis de $0,5 \mathrm{ml}$, en la glándula mamaria inguinal de la cadena derecha para todos los pacientes; en el segundo protocolo se inyectaron $0,5 \mathrm{ml}$ de la misma solución de GPDM diluida en $0,5 \mathrm{ml}$ de suero fisiológico $(\mathrm{NaCl}$ al $9 \%$ ) vía intradérmica en la glándula mamaria izquierda de todos los pacientes.

Se eligió la vía de administración intradérmica porque facilita la migración del agente de contraste en los conductos linfáticos debido al efecto de la alta presión en el tejido conectivo denso de esta zona anatómica, como afirman Suga et al. [12] y Burnand et al. [19]; y después de la aplicación del agente de contraste, se practicó un masaje de treinta segundos en el punto de aplicación para facilitar la migración del GPDM, como recomiendan Ruehm et al. [13]. Asignamos una puntación a los conductos linfáticos en función del grado de visualización del trayecto del conducto linfático en 
las imágenes del estudio, siguiendo los criterios que se reflejan en la tabla 1.

TABLA I

CRITERIOS QUE DETERMINAN LA PUNTUACIÓN ASIGNADA A LOS CONDUCTOS LINFÁTICOS DE ACUERDO CON SU VISUALIZACIÓN

\begin{tabular}{|c|l|}
\hline 0 & \multicolumn{1}{|c|}{ Conductos linfáticos no visibles. } \\
\hline 1 & Conductos linfáticos visibles solo en su inicio. \\
\hline 2 & Conductos linfáticos visibles en su tramo final. \\
\hline 3 & $\begin{array}{l}\text { Conductos linfáticos solo visibles en la mitad de su } \\
\text { trayecto. }\end{array}$ \\
\hline 4 & Conductos linfáticos visibles intermitentemente. \\
\hline 5 & Conductos linfáticos visibles en su totalidad. \\
\hline
\end{tabular}

Fuente: los autores.

\section{6 Tratamiento estadístico}

Los resultados de intensidad de señal de las diferentes estructuras estudiadas fueron expresados como media \pm desviación estándar. Para la comparación de estos valores empleamos la prueba ANOVA, proporcionando el valor del estadístico $(F)$ y su correspondiente $(p)$ se consideró que las diferencias eran estadísticamente significativas cuando $p$ fue menor a 0,05 (nivel de significancia del 95\%). Todo el análisis estadístico fue realizado con el programa Epi Info versión 6.04 [40].

\section{RESULTADOS Y DISCUSIÓN}

Validación de la técnica y metodología: el estudio comparativo de las intensidades de señal obtenidas en estas tres estructuras demostró la existencia de diferencias estadísticamente significativas entre ellas ( $F=1857,1, p<0,001)$. Cuando comparamos el promedio de la intensidad de señal entre ellas, consideradas individualmente, la diferencia entre las intensidades de señal del aire y el músculo resultó significativa ( $F=30,7, p$ $<0,001$ ); de igual forma, cuando se comparó el promedio de la IS del aire con el promedio de IS de la grasa la diferencia fue estadísticamente significativa ( $F=34,6, p<0,001)$; del mismo modo, la diferencia entre el promedio de IS del músculo y de la grasa se mostró estadísticamente significativa $(F=23,2, p<0,001)$.

La Tabla II refleja el promedio de la intensidad de señal y la desviación estándar (DS) de las mediciones realizadas en la estructuras aire, grasa y músculo.
TABLA II

PROMEDIO DE LA INTENSIDAD DE SEÑAL DE LAS ESTRUCTURAS EN ESTUDIO EN LA VALIDACIÓN DE LA TÉCNICA

\begin{tabular}{|c|c|c|}
\hline & IS media & DS \\
\hline Aire & 5,2 & 1,2 \\
\hline Músculo & 84,9 & 12,1 \\
\hline Grasa & 276,9 & 36,8 \\
\hline
\end{tabular}

Fuente: Ios autores.

\subsection{Intensidad de señal de los linfonodos inguinales en hembras del grupo I}

Se encontró que la intensidad de la señal en los linfonodos inguinales en los animales del grupo I fue, antes de la administración del contraste, de $51,3 \pm 5,4$ en el linfonodo derecho y de 50,8 $\pm 5,4$ en el linfonodo izquierdo; al comparar estos resultados podemos comprobar que no existe diferencia estadísticamente significativa $(F=0,05$, $p=0,818$ ) entre la intensidad de señal obtenida antes de la administración del contraste en los linfonodos izquierdos y derechos de los animales de este grupo, como se observa en la Fig. 2.

Fig. 2. IMÁGENES EN SERIE 3D TOF PRECONTRASTE (A) Y POSCONTRASTE (B) DE LOS LINFONODOS INGUINALES SUPERFICIALES

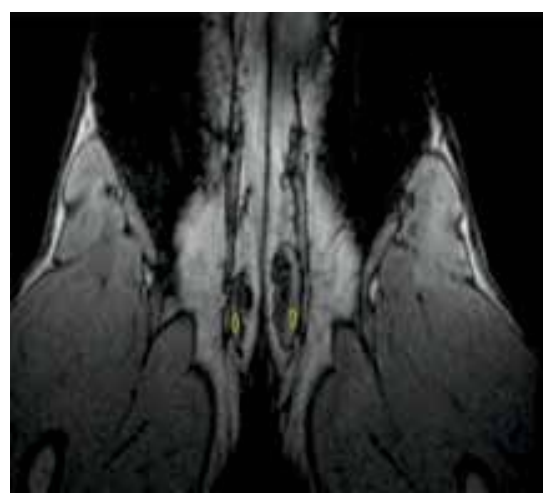

A

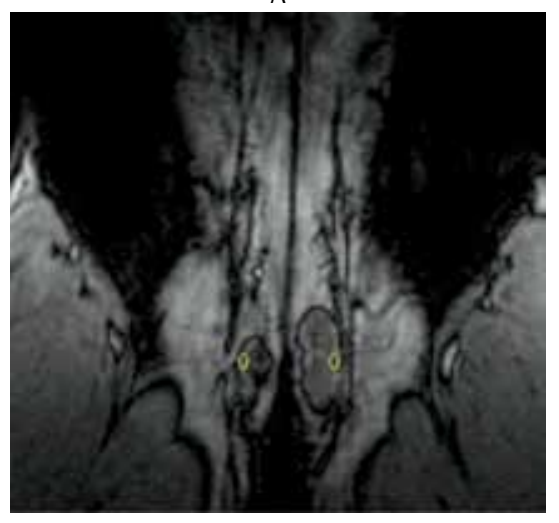

B

Fuente: Ios autores. 
Tras la inyección del agente de contraste siguiendo el protocolo I de aplicación del mismo se obtuvo un valor en la intensidad de la señal del linfonodo derecho posterior a la administración del agente de contraste de 118,2 $\pm 30,8$ como se aprecia en la Tabla III, de esta forma se pudo ver un incremento positivo del $56,7 \%$, en la intensidad de la señal con respecto a la intensidad de señal del linfonodo antes de la administración del agente de contraste, este realce en la intensidad de la señal del linfonodo resultó estadísticamente significativo $(F=7,41, p=0,020)$.

De igual forma, la administración del agente de contraste conforme al protocolo II arrojó un valor en la intensidad de la señal del linfonodo izquierdo posterior a la administración del agente de contraste de 110,6 \pm 29,6 (Tabla II, mostrando un incremento positivo del $54,1 \%$ en la intensidad de la señal con respecto a la intensidad de la señal del linfonodo antes de la administración del agente de contraste, que como en el caso anterior fue estadísticamente significativo ( $\mathrm{F}=$ $6,28, p=0,024)$.

Si comparamos el uso de los dos protocolos de administración del agente de contraste, teniendo en cuenta la intensidad de señal que se observa tras la administración del gadolinio, encontramos que no hubo diferencia significativa entre ellos ( $F$ $=0,38, p=0,541$ ).

TABLA III

PROMEDIOS DE INTENSIDAD DE SEÑAL EN LINFONODOS DE HEMBRAS DEL GRUPO I

\begin{tabular}{|l|l|l|l|l|}
\hline & LDpre & LDpost & LIpre & LIpost \\
\hline Promedio & 51,3 & 118,2 & 50,8 & 110,6 \\
\hline DS & 5,4 & 30,8 & 5,4 & 29,6 \\
\hline
\end{tabular}

LDpre $=$ promedio intensidad de la señal del linfonodo derecho antes del contraste. LDpost $=$ promedio intensidad de la señal del linfonodo derecho posterior al contraste. Llpre = promedio intensidad de la señal del linfonodo izquierdo antes del contraste. LIpost = promedio intensidad de la señal del linfonodo izquierdo posterior al contraste.

Fuente: los autores.

\subsection{Intensidad de señal de los linfonodos de pacientes con procesos patológicos en la glándula mamaria en IRM grupo II}

El estudio de las imágenes de los linfonodos de las hembras de nuestro grupo II de trabajo, obtenidas mediante RM en serie 3D TOF sin con- traste, reveló para estas estructuras una intensidad de señal que se mantuvo en un rango de 46,9 a 115,8 , con un valor medio de $65,7 \pm 18,5$. En el linfonodo inguinal derecho, osciló de 37,6 a 115,8 , con una media de $66,7 \pm 20,8$, mientras que en el linfonodo izquierdo la intensidad de señal varió de 46,9 a 102.1, con un valor promedio de $64,8 \pm 16,1$, sin diferencias significativas entre ambas, como tampoco se encontró diferencia entre cualquiera de estas últimas y la intensidad de señal de los linfonodos de ambas cadenas considerados conjuntamente.

Cuando analizamos el promedio de las intensidades de señal en las IRM de los linfonodos de las hembras de este grupo, afectadas por procesos patológicos en sus mamas, se apreció un aumento en la intensidad de señal con respecto a la intensidad de señal promedio de los linfonodos de hembras sanas como se observa en la tabla IV.

TABLA IV

PROMEDIO DE LA INTENSIDAD DE SEÑAL DE LOS LINFONODOS ANTES DEL CONTRASTE DE LOS PACIENTES CON PROCESOS PATOLÓGICOS EN LA GLÁNDULA MAMARIA

\begin{tabular}{|c|c|c|c|c|}
\hline & XLANDPS-II & XLANIZPS-II & XLDPRE-I & XLIPRE-I \\
\hline $\begin{array}{c}\text { Promedio } \\
\text { intensidad } \\
\text { de señal }\end{array}$ & 66,7 & 64,8 & 51,3 & 50,8 \\
\hline $\begin{array}{c}\text { Desviación } \\
\text { estándar }\end{array}$ & 20,8 & 16,1 & 5,4 & 5,4 \\
\hline
\end{tabular}

XLANDPRE $=$ linfonodo derecho en precontraste, XLANIZQPRE $=$ linfonodo izquierdo en precontraste. Pacientes sin procesos patológicos en la glándula mamaria: XLDPRE-I = linfonodo derecho en precontraste grupo I, XLIPREII = linfonodo izquierdo en precontraste, grupo I.

Fuente: los autores.

Como se puede observar, el promedio de la intensidad de señal del linfonodo derecho en las imágenes obtenidas antes de la administración del contraste en los pacientes con procesos patológicos en glándula mamaria es un 23,1\% mayor que el de los linfonodos de las hembras sin procesos patológicos en dichas glándulas, si bien esta diferencia no resulta estadísticamente significativa $(F=2,27, p=0,163)$; como tampoco lo es la diferencia entre el valor medio de IS que ofrecieron las imágenes de los linfonodos de las hembras sanas y el valor medio de este mismo parámetro medido sobre el linfonodo izquierdo de las hembras afectadas por procesos patológicos, aunque dicha IS resultara en estas un $21,5 \%$ mayor que en aquellas $(F=2,62, p=0,136)$. 
Cabe destacar que el análisis histopatológico de los linfonodos recogidos reveló la presencia de células tumorales en los vasos linfáticos de una hembra de este grupo de estudio con sospecha de micrometástasis en una de las secciones histológicas. En este caso, el promedio de la intensidad de señal del linfonodo implicado, en las IRM anteriores a la inyección del contraste, presentó un incremento positivo del $50,2 \%$ en relación con los linfonodos de los pacientes del grupo I; de igual forma, se detectó un incremento positivo de la intensidad de señal en las imágenes de dicho linfonodo obtenidas tras la administración del contraste de un $17,9 \%$ con respecto a la IS de las imágenes obtenidas tras la administración del contraste en las hembras del grupo I como se puede apreciar en la Fig. 3.

Hemos de hacer hincapié en el hecho de que el estudio mediante imagen por resonancia magnética ya demuestra la existencia de una alteración en el linfonodo compatible con la metástasis que posteriormente confirmaría el diagnóstico histopatológico, que determina la presencia de dicha metástasis precisamente en la localización señalada por el estudio mediante IRM.

Fig. 3. LINFONODO CON PRESENCIA DE NUMEROSOS ÉMBOLOS DE CÉLULAS NEOPLÁSICAS OCUPANDO LA LUZ DE VASOS AFERENTES $(\rightarrow)$

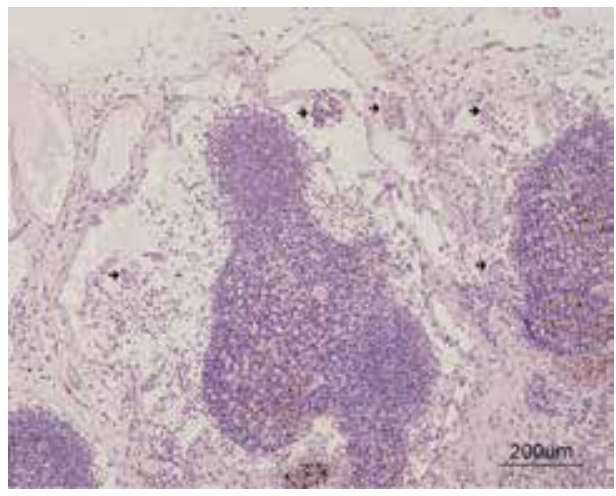

Fuente: los autores.

De igual forma, el estudio histopatológico de las muestras obtenidas en otra hembra del grupo II mostró, mediante la técnica de inmunohistoquímica, la presencia ocasional de émbolos tumorales en vasos linfáticos, sin implantación en ninguna de las secciones examinadas. En este caso, el promedio de la intensidad de señal en las imágenes de RM del linfonodo previas a la inyección del contraste resultó un $36,8 \%$ superior a la media de IS de los linfonodos de pacientes sanos.
En este mismo caso, tras la administración del contraste se detectó en las IRM un aumento de la intensidad de señal de 3,6\% con respecto a la IS de las imágenes obtenidas tras la administración del contraste en las hembras sanas, del grupo I, lo cual se refleja en la Fig. 4.

También en este caso las imágenes obtenidas mediante resonancia magnética revelaron en el linfonodo alteraciones compatibles con la presencia de una metástasis que fue posteriormente confirmada mediante el diagnóstico histopatológico.

Fig. 4. PRESENCIA DE ESCASOS ÉMBOLOS DE CÉLULAS TUMORALES EN VASOS LINFÁTICOS (è), IDENTIFICADOS MEDIANTE TÉCNICA INMUNOHISTOQUÍMICA (ABC-PEROXIDASA)

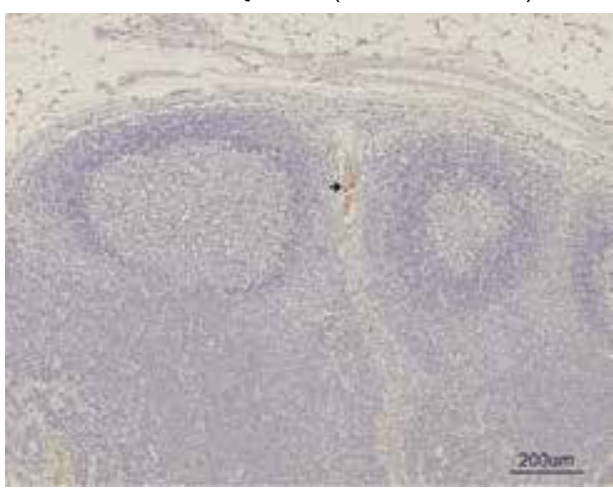

Fuente: los autores.

Al considerar únicamente el estudio de las imágenes obtenidas de los linfonodos cuyo estudio histopatológico posterior reveló la presencia de émbolos tumorales, se puede comprobar que el promedio de la intensidad de señal en dichas imágenes previas a la administración del contraste resultó un $44,1 \%$ superior a la intensidad de señal media de las imágenes obtenidas, asimismo, antes de la aplicación de contraste, de los linfonodos de animales sin problemas patológicos en las glándulas mamarias, aunque esta diferencia no resulta estadísticamente significativa $(F=3,83$, $P=0,301)$. Tras la administración del contraste, las imágenes de estos linfonodos afectados por procesos metastásicos mostraron un aumento de la intensidad de señal del $11,1 \%$ con respecto al promedio de intensidad de señal de los linfonodos del grupo I, también tras administración de contraste, diferencia que, como en el caso anterior, no resulta significativa $(F=0,69 . p=0,41)$.

En cuanto al caso de la masa de carácter benigno diagnosticada en el grupo II de nuestro estudio (Hiperplasia mamaria con ectasia ductal), las imágenes del linfonodo correspondiente previas a 
la inyección de contraste mostraron una la intensidad de señal media aumentada tan solo en un $12,5 \%$ con respecto a la IS de las imágenes de resonancia magnética obtenidas antes de la inyección del contraste en las hembras sanas.

Al igual que en los pacientes del grupo I, a los animales de este grupo se les inyectó el agente de contraste siguiendo el protocolo I de administración en el linfonodo derecho, tras lo cual se realizó el estudio de las características de la intensidad de la señal en las series así obtenidas, que revelaron una intensidad de señal media, para los citados linfonodos derechos, de 129,5 $\pm 65,5$, es decir, un $8,7 \%$ superior a la intensidad de la señal media de las imágenes poscontraste de los linfonodos del grupo I, diferencia estadísticamente no significativa $(F=0,50, p=0,493)$.

Del mismo modo, al comparar los promedios de los valores de intensidad de señal de las imágenes de resonancia magnética obtenidas en los linfonodos linfáticos izquierdos tras la administración del agente de contraste, en este caso siguiendo el protocolo II, encontramos que dicho valor promedio resulta para los animales del grupo II de $130,6 \pm 65,7$, es decir, un 15,3\% superior al de los del grupo I, aunque tampoco en este caso la diferencia resulta estadísticamente significativa $(F=0,89, p=0,364)$.

\subsection{Visualización de los conductos linfáticos en hembras sanas}

En nuestro estudio de las IRM observamos que no todos los conductos mostraban un trayecto homogéneo, y que en algunos casos solo la administración del agente de contraste facilitó la identificación de estas estructuras, como se observa en al Fig. 5.

En los animales sin procesos patológicos en la glándula mamaria (grupo I de nuestro estudio), el porcentaje que la visualización del conducto linfático derecho mejoró con la administración del agente de contraste, siguiendo el protocolo I, fue del $75,0 \%$, si bien debemos tener en cuenta que el porcentaje restante $(25,0 \%)$ corresponde tanto a animales en los cuales el conducto era visible en su totalidad $(8,3 \%)$ como parte de aquellos en los que los conductos que eran visibles de forma intermitente (16,7\%). En estos mismos animales, la visualización del trayecto de los conductos lin- fáticos izquierdos en las imágenes obtenidas tras la administración del agente de contraste según el protocolo II mejoró, con respecto a las series previas en el $66,7 \%$ de los casos, correspondiendo también en este caso el porcentaje restante a las imágenes de los casos en los que los conductos linfáticos eran visibles de forma intermitente $(16,6 \%)$ o en su totalidad (16,6\%) en las IRM obtenidas previamente a la administración del contraste.

Fig. 5. VISUALIZACIÓN DE LOS CONDUCTOS LINFÁTICOS EN SU TOTALIDAD, ANTES DEL CONTRASTE (A) Y POSTERIOR AL CONTRASTE (B)

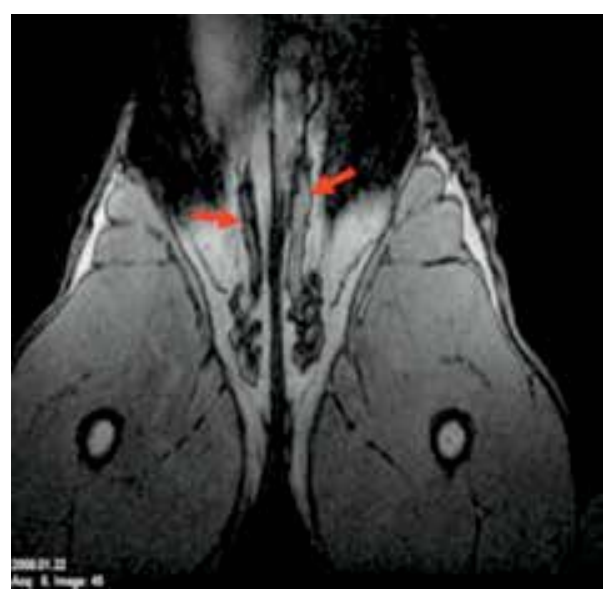

A

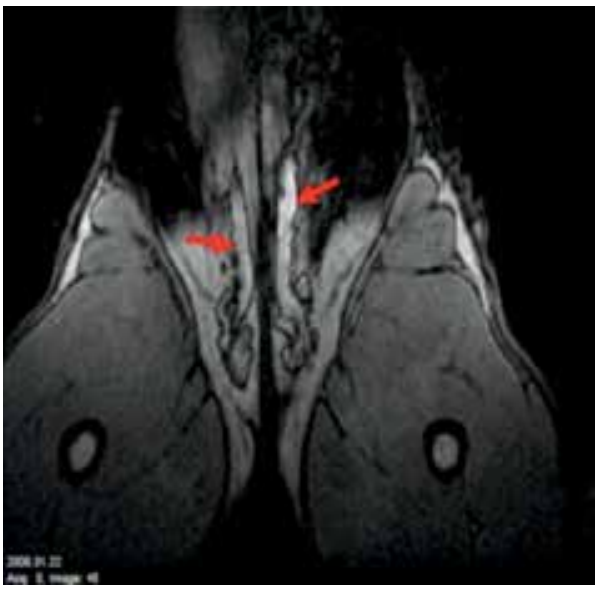

B

Fuente: Ios autores.

Los valores medios obtenidos en nuestro estudio en cuanto a la visualización de los conductos linfáticos en hembras sin procesos patológicos en la glándula mamaria, tanto en las imágenes anteriores a la administración del contraste como en las posteriores a dicha administración siguiendo los protocolos I y II, se reflejan en la Tabla V. 
TABLA V

DESCRIPCIÓN DE LA VISUALIZACIÓN DE LOS CONDUCTOS LINFÁTICOS EN PACIENTES SIN PROCESOS PATOLÓGICOS EN LA GLÁNDULA MAMARIA, PROMEDIO DE LOS PUNTOS DE LOS CONDUCTOS LINFÁTICOS

\begin{tabular}{|l|l|l|l|l|}
\hline & CDP & CIP & CDPOS & CIPOS \\
\hline Promedio & 3,1 & 3,3 & 4,4 & 4,5 \\
\hline DS & 1,4 & 1,4 & 0,7 & 0,7 \\
\hline
\end{tabular}

Fuente: los autores.

CDP = conducto linfático de la cadena derecha antes del contraste. CIP = conducto linfático de la cadena izquierda antes del contraste. CDPOS $=$ conducto linfático de la cadena derecha posterior al contraste. CIPOS = conducto linfático de la cadena izquierda posterior al contraste.

Encontramos que los valores medios en cuanto a la visualización de los conductos linfáticos en animales sin procesos patológicos en la glándula mamaria fueron, antes de la administración del agente de contraste, de $3,1 \pm 1,4$ en el conducto linfático derecho y de 3,3 $\pm 1,4$ para el conducto linfático izquierdo; al comparar estos resultados podemos comprobar que no existe diferencia estadísticamente significativa $(F=0,12, p=0,729)$.

Cuando se administró el agente de contraste se obtuvo un valor promedio en la visualización de los conductos de 4,4 \pm 0,7 para el conducto linfático derecho, al que se administró el contraste conforme al protocolo I, y de 4,5 $\pm 0,7$ para el conducto izquierdo, al que se administró el contraste conforme al protocolo II; al comparar estos resultados podemos ver que no existe diferencia estadísticamente significativa $(F=0,12, p=0,729)$.

\subsection{Visualización de los conductos linfáticos en hembras con procesos patológicos en glándula mamaria}

La visualización de los conductos linfáticos en las imágenes de IRM obtenidas en pacientes con procesos patológicos en la glándula mamaria, conforme a la valoración numérica descrita en el apartado de Material y Métodos, fue, antes de la administración del agente de contraste, de 2,0 $\pm 1,7$ para el conducto linfático derecho y de $0,5 \pm 1,0$ en el conducto izquierdo; cuando comparamos estos resultados podemos observar que existe diferencia estadísticamente significativa ( $F$ $=5,78, p=0,025)$.

Una vez administrado el agente de contraste, se obtuvo, para la visualización de los citados conductos en las IRM, un valor promedio de 3,7 $\pm 0,8$ para el conducto linfático derecho, al que siguiendo el protocolo I se había inyectado por vía intradérmica el gadopentetato de dimeglumina sin diluir, y de 2,5 \pm 1,9 para el conducto linfático izquierdo, al que se había inyectado por la misma vía el contaste diluido, conforme al protocolo II de administración; al comparar estos resultados podemos observar que no existe diferencia estadísticamente significativa ( $F=1,84, p=0,19)$ Tabla VI.

TABLA V

DESCRIPCIÓN DE LA VISUALIZACIÓN DE LOS CONDUCTOS LINFÁTICOS EN PACIENTES CON PROCESOS PATOLÓGICOS EN LA GLÁNDULA MAMARIA, PROMEDIO DE LOS PUNTOS DE LOS CONDUCTOS AFECTADOS

\begin{tabular}{|l|l|l|l|l|}
\hline & CDP & CIP & CDPOS & CIPOS \\
\hline Promedio & 2,0 & 0,5 & 3,7 & 2,5 \\
\hline DS & 1,7 & 1,0 & 0,8 & 1,9 \\
\hline
\end{tabular}

Fuente: los autores.

$\mathrm{CDP}=$ conducto linfático de la cadena derecha en precontraste. $\mathrm{CIP}=$ conducto linfático de la cadena izquierda en precontraste. CDPOS = conducto linfático de la cadena derecha en poscontraste. CIPOS = conducto linfático de la cadena izquierda en poscontraste.

\section{5 Discusión}

Para llevar a cabo la validación de la técnica y de la metodología empleadas en lo referente a mediciones realizadas mediante la planimetría manual, optamos por las IRM ponderadas en T1, al ser estas las que nos ofrecieron mayor calidad y definición para el trazado manual de las diferentes áreas por estudiar, considerando siempre la mejor visualización de la estructura en estudio por encima de la eficacia que en el diagnóstico clínico nos podía aportar la imagen, ya que, como se ha demostrado [38], [39], [41], el manejo de las secuencias de pulsos hace prevalecer determinada información mediante la potenciación de la imagen. Coincidimos así con los autores de numerosos estudios que describen el empleo de técnicas de planimetría manual, utilizando el mismo tipo de imágenes, tanto en trabajos desarrollados con equipos de resonancia magnética de bajo campo (0,2 Teslas) [37], [39], [42], [43], como es el caso de nuestro estudio, como en investigaciones lleva- 
das a cabo con equipos de resonancia magnética de alto campo (1,5 a 2 Teslas) [44]-[49].

La elección de las tres estructuras (aire, grasa y músculo) de las que se obtendrían, en nuestras imágenes por resonancia magnética ponderadas en T1, los valores medios de intensidad de señal que nos habrían de permitir crear un patrón de IS para cada estructura y diferenciarlas así de otras estructuras de interés en este estudio, se hizo de acuerdo con el hecho, reafirmado en numerosos trabajos de investigación [35]-[39]. que en las imágenes ponderadas en T1 la grasa proporciona la máxima intensidad de señal (que se traduce en el blanco más intenso) y la señal que aporta el aire es la mínima (por lo que ofrece un color negro), mientras que la imagen del músculo resulta con una señal intermedia entre las dos anteriores, a consecuencia de la relación entre la densidad de los núcleos de hidrógeno de estas estructuras y el entorno bioquímico en el que se encuentran, así como de las secuencias de pulsos que tienen lugar en esta potenciación de la imagen, como se ha descrito anteriormente [38], [39], [41].

La baja intensidad de la señal que obtuvimos al trazar una ROI de $12 \mathrm{~mm}^{2}$ en el área correspondiente al aire en todas la IRM del estudio $(5,2$ $\pm 1,2$ ) se explica así por la ausencia de tejido en esta zona, de modo que la baja densidad, e incluso ausencia prácticamente total de núcleos de hidrógeno, determina la drástica reducción de la intensidad de señal detectada al emplear la herramienta del programa informático mencionada para tal efecto, como queda también reflejado en las publicaciones revisadas en las que se afirma que, en las imágenes ponderadas en T1, el aire se verá más negro al determinar la menor intensidad de señal [38], [39], [50]-[52].

En nuestro estudio, la IS de la grasa en las IRM de las hembras sin procesos morbosos en la glándula mamaria, medida de nuevo en un trazo de 12 $\mathrm{mm}^{2}$ sobre la estructura correspondiente, resultó ser de 276,9 $\pm 36,8$. Estos datos concuerdan con lo descrito por Gili [38], Jaramillo et al. [39], Dennis [50], Snellman [51] y Thrall [52], que reportan que la grasa en imágenes ponderadas en T1 se aprecia como una estructura brillante debido a su elevada intensidad de señal.

De igual forma, tras trazar una ROI de $12 \mathrm{~mm}^{2}$ para la determinación de las características del músculo en las IRM de nuestro estudio, obtuvi- mos una intensidad de señal de $84,9 \pm 12,1$; valor que coincide con lo reportado por la bibliografía [38], [39], [50]-[52], en trabajos que establecen que la intensidad de señal del músculo es intermedia, determinando en la imagen un tono que se sitúa en la mitad de una escala de grises.

Al realizar el estudio comparativo de los resultados de la valoración de la IS en estas tres estructuras comprobamos que las diferencias entre ellas, tanto tomadas conjuntamente como comparadas de dos en dos, resultaron altamente significativas, coincidiendo con las afirmaciones de Gili [38], Jaramillo et al. [39], Dennis [50], Snellman [51] y Thrall [52], que definen la grasa como hiperintensa, el músculo como hipointenso y el aire negro en una imagen ponderada en T1, lo que refuerza nuestra decisión de tomar estos parámetros como patrón de comparación frente a las demás estructuras en estudio.

Intensidad de señal de los linfonodos y características de los conductos linfáticos del grupo de animales sanos: como quiera que son numerosas las publicaciones con base en las cuales se puede considerar en exceso variable la especificidad de la RM potenciada con la administración de gadopentetato de dimeglumina [53]-[56], [60]. Hemos intentado dilucidar, con el presente estudio, si el modo de administración de este contraste determinaba o no variaciones en los resultados de captación, ya que no cabe duda de que, en el caso de las IRM de mama, la potenciación de la imagen con este agente de contraste permite detectar, con elevada sensibilidad, alteraciones neoplásicas, como han demostrado Nunes et al. [57], Orel et al. [58], y Kinoshita [59]. Seleccionamos para ello la vía intradérmica, ya que, como indican Kern [18] y Tanis et al. [16], el origen embrionario común de la glándula mamaria y la piel que la recubre, sumado a la confluencia del drenaje linfático de ambas estructuras mamarias en el plexo subdérmico, permiten predecir la utilidad de esta vía de inyección para tal fin.

Las imágenes de los linfonodos y conductos linfáticos obtenidas en nuestro estudio mediante RM antes y después de la inyección del gadopentetato de dimeglumina siguiendo los citados protocolos nos permiten concluir que la aplicación intradérmica del agente de contraste resulta eficaz en la visualización del primer relevo ganglionar (linfonodo inguinal superficial) también en anima- 
les sin procesos patológicos en la glándula mamaria, como determinaron Kern [18] y Tanis et al. [16], en su caso para el cartografiado del linfonodo centinela en tumores mamarios en humanos.

Los resultados en cuanto a las características de intensidad de señal de las IRM de los linfonodos inguinales de las perras que integraban el grupo II de trabajo, obtenidas en series potenciadas en T1 antes de la administración del contraste $(65,7 \pm 18,5)$, muestran en todos los casos una hiperintensidad relativa con respecto a la IS de los linfonodos de las IRM del grupo I, integrado por hembras sanas, en las mismas series $\mathrm{T} 1$ previas a la administración del contraste; sin embargo, este aumento en la IS (del 23,1\% en el lado derecho y del $21,5 \%$ en el lado izquierdo) no resultó estadísticamente significativa $(F=2,27, p=1,63$ y $F=$ $2,62, p=0,136$, respectivamente).

En nuestra opinión, estas diferencias en cuanto a la IS de las IRM de ambos grupos de trabajo (hembras sanas y hembras con procesos mamarios) probablemente estén relacionadas con el hecho de que algunos de los linfonodos de las hembras del grupo II estarían recibiendo la linfa procedente de glándulas mamarias que cursaban en ese momento con procesos patológicos activos, por lo que podría ser portadora de células tumorales, en las que el entorno bioquímico se ve alterado, modificando así, como indican Desgrez et al. [41] y Gili [38], la información relacionada con estas células que se obtiene para cada vóxel durante la etapa de relajación en el proceso de construcción de la IRM.

Así, considerando en nuestras IRM el promedio de intensidad de señal únicamente de los linfonodos cuyo análisis histopatológico posterior reveló alteraciones metastásicas, podemos constatar, en las IRM obtenidas antes de la administración del contraste, un incremento positivo en el promedio de intensidad de señal del $44,1 \%$ con respecto al promedio de IS de los linfonodos del grupo I, en la misma serie. Achacamos la ausencia de significación estadística en esta diferencia únicamente al tamaño de la muestra, si bien nos parece que la alteración en la IS de las imágenes de los linfonodos regionales en pacientes con mamas afectadas por procesos patológicos con respecto a la IS del tejido ganglionar sano, sería un buen indicador para determinar mediante IRM el linfonodo centinela en pacientes con neoplasias mamarias, pues a partir de un porcentaje dado de incremento en la intensidad de señal del linfonodo afectado, cuya magnitud habría de ser determinada con un número mayor de casos, resultaría indicador de metástasis en dicho linfonodo.

De la validez del diagnóstico imaginológico no solo en la determinación del linfonodo centinela, sino también en la detección de alteraciones metastásicas de las neoplasias mamarias da fe el hecho de que, en nuestro estudio, todas las afectaciones ganglionares confirmadas por el estudio histopatológico de las muestras ganglionares habian sido previamente detectadas en las imágenes de resonancia magnética como sospechosas de alteración por un marcado incremento en la intensidad de señal con respecto al tejido ganglionar sano.

Por otra parte, llama la atención que cuando se administró el agente de contraste, la diferencia en el realce obtenido entre las cadenas linfáticas derecha e izquierda $(8,7 \%$ en los linfonodos derechos, $15,3 \%$ en los izquierdos) que, aun resultando no significativa, podría sugerir que con una casuística más elevada tal vez se alcanzara la significación estadística. En este sentido, podemos apuntar, sin pretender sacar de ello conclusión alguna, debido a la escasa incidencia en nuestro estudio, que fue precisamente en la cadena ganglionar izquierda, cuya media de realce casi duplica al que se produce en la derecha, en la que se detectó mediante estudio histopatológico el caso de émbolos tumorales con micrometástasis ganglionar. De hecho, en este caso, el incremento positivo de la IS en la IRM obtenida tras la inyección del contraste, con respecto al observado en las IRM correspondientes del grupo I, fue de un $17.9 \%$.

En cuanto a la visualización de los conductos linfáticos en pacientes con procesos patológicos en la glándula mamaria coincidimos con los autores consultados (Viale et al. [32]; Wilhelm [33]; Mariani [34]) en destacar la importancia de la visualización del trayecto que discurre desde el lugar de inyección del agente de contraste hasta el primer relevo ganglionar en el cartografiado del conducto linfático y del linfonodo al que drena, de cara a la identificación del Linfonodo Centinela y en nuestro caso, el estudio de las neoplasias mamarias. Encontramos así en el estudio de las IRM de nuestros pacientes del grupo II que, en cuanto 
a su morfología, el trayecto del conducto linfático mostraba un aspecto sinuoso e intermitente en las imágenes potenciadas en T1, si bien en algunos casos no era posible identificar en ellas el citado conducto linfático.

Valorada la posibilidad de visualización del trayecto linfático con la ayuda de la misma tabla de puntuación descrita para el grupo I de trabajo, nuestros resultados, que muestran una gran variabilidad en la capacidad de cartografiado de dichos trayectos (promedio de 2,0 $\pm 1,7$ para el conducto linfático derecho antes de la administración del contraste, 3,7 . $\pm 0,8$ después de dicha administración; 0,5 \pm 1,0 para el conducto linfático izquierdo antes de la administración del contraste, 2,5 $\pm 1,9$ después), concuerdan con los resultados de las investigaciones de Suga et al. [12] y Mitsumori et al. [60] si bien ellos utilizaron gadolinio como agente de contraste para facilitar la visualización del conducto linfático en pacientes con procesos patológicos en la glándula mamaria.

En nuestro caso, la capacidad de visualizar el trayecto de los conductos linfáticos de los pacientes aquejados de procesos mamarios en las IRM obtenidas antes de la inyección del agente de contraste mostró una diferencia estadísticamente significativa entre los conductos linfáticos derecho $(2,0 \pm 1,7)$ e izquierdo $(0,5 \pm 1,0)$. Únicamente podemos relacionar esta diferencia con el hecho de que, en nuestro grupo II de estudio, el $60,0 \%$ de los procesos morbosos diagnosticados se asentaban en glándulas mamarias de la cadena derecha, y únicamente el 40,0\% restante se localizaba en una u otra glándula de la cadena mamaria izquierda.

Una vez administrado el agente de contraste, sin embargo, la diferencia entre la posibilidad de visualizar, en las IRM, el trayecto derecho $(3,7 \pm$ $0,8)$ e izquierdo $(2,5 \pm 1,9)$ no resultó significativa.

\section{CONCLUSIONES}

El empleo de un equipo de resonancia magnética de bajo campo permite identificar mediante la intensidad de señal los linfonodos que recogen linfa de procesos oncológicos provenientes de las glándulas mamarias respecto de los linfonodos sanos, lo cual ayuda a determinar estrategias quirúrgicas en pacientes que cursan procesos oncológicos en glándula mamaria en la especie canina. De igual forma, con esta técnica se logró visualizar el trayecto de los conductos linfáticos que drenan linfa desde el proceso oncológico en esta especie.

\section{AGRADECIMIENTOS}

El autor quiere expresar su agradecimiento a la doctora Alicia Ester Serantes Gómez por todo su apoyo e interés científico durante la realización de este trabajo, ya que sin su orientación y dedicación no hubiese culminado este estudio. De igual forma quiero agradecer a todo el equipo que me colaboró durante la realización de los protocolos de anestesia y adquisición de imágenes muy especialmente al doctor José Manuel Gonzalo Orden y a la doctora Marta Reguiero Purriños. Por último, y no menos importante, quiero expresar mi agradecimiento por todo su acompañamiento durante el desarrollo del estudio a los doctores Jesús Sánchez García, Alexandre Duarte, Francisco Jiménez, a la doctora Johanna Fonseca y a todos los que quisieron aportar durante el desarrollo de este estudio en la Unidad de Cirugía y Diagnóstico por Imagen de la Facultad de Veterinaria de la Universidad de León.

\section{REFERENCIAS}

[1] D. Slatter, Manual de cirugía de pequeños animales, 1997, Cáp. 148, pp. 776-782.

[2] C.T. Pereira, S.C. Rahal, D.E. Carvalho, J.C. Baleiro, A. Ribeiro, "Lymphatic drainage on healthy and neoplasic mammary glands in female dogs: can it really be altered?", Anat. Hembryol, vol. 32, pp. 282-290.

[3] D. W. Fawcett, Tratado de histología. Sistemas vasculares sanguíneo y linfático. Interamericana McGraw Hill, 1994, p. 404.

[4] R. Barone, "Anatomia comparata dei mammiferei domestici. Splancnologia", Ed agricole, vol. 4. pp. 404406, 1994.

[5] E. Ayllon, A.J. Flores, Caracterización de tumores mamarios en la perra. Curso Oncología Mamaria en la perra, Torremolinos (Málaga), 1996. http: //www. Veterinaria.org/ajfa/col I.htm.

[6] P.E. Flores, U.G. Cattaneo, "Tumores mamarios en caninos domésticos, epidemiología, criterios de diagnóstico y enfoque terapéutico". Monografías de medicina veterinaria, col. 21 jul. 2001. 
[7] L. Marconato, F. Del Piero, Oncologia medica dei piccoli animali, Poletto Editore Srl., 2005, pp. 2-16, 47$50,440-459,670-676,679.681$.

[8] M. Weiss, R.G. Baumeister, K. Hahn, "Dynamic iymph flow imaging in patients with edema of the lower limb for evaluation of the functional outcome after autologous lymph vessel transplantation: an 8-year follow-up study", Eur J Nucl Med Mol Imaging., 30, pp. 202-6, 2003.

[9] Cserni, "Metastases in axillary sentinel lymph nodes in breast cancer as detected by intensive histopathological work up", J Clin Pathol. 52, pp. 922-4, 1999.

[10] R. El Khouli, A. Jacobs, D. Bluemke, "Magnetic resonance imaging of the breast", Seminars in Roentgenology, Elservier, 2008.

[11] F. Rety, O. Clement, N. Siauve, "MR lymphography using iron oxide nanoparticles in rats: Pharmacokunesis in the lymphatic system after intravenous injection", J Magn Reson Imaging., 12, pp. 734-9, 2000.

[12] K. Suga, Y. Yuan, N. Ogasawara, M. Okada, N. Matsunaga, "Visualization of normal and interrupted lymphatic drainage in dog whit interstitial MR Lymphography using an extracellular MR Contrast Agent, Gadopentetate dimeglumine", Investigative radiology, vol 38, No. 6, Jun. 2003

[13] S.G. Ruehm, C. Corot, J.F. Debatin, "Interstitial MR lymphography with a conventional extracellular gadolinium-based agent: assessment in rabbits", Radiology, 218, pp. 664-669, 2001.

[14] S.G. Ruehm, T. Schroeder, J.F. Debatin, “Interstitial MR Lymphography with gadoterate meglumine: initial experience in humans", Radiology, 220, p. 816, 2001.

[15] B.R. Krynyckyi, M.K. Shafir, S.C. Kim, D.W. Kim, A. Travis, R.M. Moadel, Ch.K. Kim. "Lymphoscintigraphy and triangulated body marking for morbidity reduction during sentinel node biopsy in breast cancer", Int Semin Surg Oncol., 2, p. 25, 2005.

[16] P.J. Tanis, O.E. Nieweg, R.A. Valdés-Olmos, E. Rutgers, B.B.R. Kron. "Anatomy and physiology of lymphatic drainage of the breast from the perspective of sentinel node biopsy", Jam Coll Surg., 192, pp. 399-409, 2001.

[17] J.F. Gallegos-Hernández, M. Aranda-Soto, P. Pichardo, R. García, J.F. Chávez, M. Arias, O.A. Melhado, “Linfadenectomía radiogiada de ganglio centinela en cáncer de mama", Cir Ciruj., vol. 5, pp. 339-345, 2002.

[18] K.A. Kern, "Sentinel Lymph node mapping in breast cancer using subareolar injection of blue dye", J Am Coll Surg., 189, pp. 539-545, 1999.
[19] K.G. Burnand, C. Mcguinness, N.R. Lagattolla, "Value of isotope lymphography in the diagnosis of lymphedema of the leg”, Br I Surg., 89, pp. 74-78, 2002

[20] D. Lubach, W. Ludemann, V. Berens, "Recent findings on the angioarchitecture of the lymph vessel system of human skin", Br. J. Dermatol, 135, p. 733, 1996.

[21] P.J. Borgstein, S. Meier, R. Pipers, “Intradermal blue dye to identify sentinel lymph node in breast cancer", Lancet, 349, pp. 1668-69, 1999.

[22] D. C. Conor, "The sentinel node in breast cancer: an update", Cancer Imaging, 5, S3-S9, 2005. DOI: 10.1102/1470-7330.2005.0039.

[23] H.S. Cody, P.I. Borgen, "State of the art approaches to sentinel node biopsy for breast cancer. Study design, patient selection, technique, and quality control at Memorial Sloan Kettering", Cancer Center. Surg. Oncol., 8, p. 85, 1999.

[24] T. Rosenow, C. Biedendieck, H. Fricke, M. Brinkmann, U. Cirkel, W.-D. Reinbold, and E. Fricke, "Sentinel Lymph Node Biopsy in Patients with Breast Cancer: Comparison of Peritumoral and Periareolar Injection", Geburtshilfe Frauenheilkunde, 72(11), pp. 1024-1028, Nov. 2012. doi: 10.1055/s-0032-1315304.

[25] A. Chagpar, R.C. Martin, C. Chao, S.L. Wong, M.J. Edwards, T. Tuttle, K.M. McMasters, "Validation of subareolar and periareolar injection techniques for breast sentinel lymph node biopsy". Arch Surg. Jun. 139 (6), 2004, pp. 614-8, discussion 618-20.

[26] S.M. Moghimi, "Bonnemain, Subcutaneous and intravenous delivery of diagnostic agents to the lymphatic system: applications in lymphoscintigraphy and indirect lymphography", Adv Drug Deliv Rev., vol. 37, pp. 295-312, 1999.

[27] G.W. Schmid-schonbein, "Microlymphatics and lymph flow”, Physiol Rev., 70, pp. 987-1028, 1990.

[28] G.L. McIntire, E.R. Bacon, K.J. Illig, "Time course of nodal enhancement with CT X-ray nanoparticle contrast agents: effect of particle size and chemical structure", Invest Radiol., 35, pp. 91-6, 2000.

[29] G. Staatz, C.C. Nolte-Erusting, G.B. Adam, “Interstitial T1-weighted MR lymphography: lipophilic perfluorinated chelotes in pigs", Radiology 220, pp. 129-134, 2001.

[30] B. Misselwitz, H. Schmitt-Willich, M. Michaelis, "Interstitial magnetic resonance lymphography using a polymetric T1 contrast agent. Initial experience with gadmer-17", Invest Radiol., 37, pp. 146-151, 2002.

[31] L. Qing, X. Jianrong, L. Ningfei, “Chronic lower extremity lymphedema: A comparative study of high-resolution interstitial MR lymphangiography and heavily 
T2-weighted MRI", European Journal of Radiology, 73, pp. 365-373, 2010.

[32] G. Viale, S. Bosari, G. Mazzarol, V. Galimberti, A. Luini, P. Veronesi, "Intraoperative examination of axillary sentinel lymph nodes in breast carcinoma patients", Cancer, 85, pp. 2433-38, 1999.

[33] A.J. Wilhelm, G.S. Mijnhout, F.J.F. Franssen, "Radiopharmaceuticals in sentinel lymph-node detection; an overview", Eur. J. Nucl. Med., 26, p. S36, 1999.

[34] G. Mariani, L. Moresco, G. Viale, "Radioguided sentinel lymph node biopsy in breast cancer surgery", J. Nucl. Med., 42, p. 1198, 2001.

[35] L. Millán, “Aplicación de la imagen por resonancia magnética al estudio de las patologías que afectan a la columna vertebral del perro", (Tesis Doctoral). Facultad de Veterinaria. Universidad de León. León. España. 2000.

[36] R. Godoy, "Diagnóstico de tumores cerebrales primarios en caninos mediante imagen por resonancia magnética", (Tesis Doctoral). Facultad de Veterinaria. Universidad de León. León, España. 2005.

[37] A.M. Duarte, "Estudio mediante resonancia magnética del cerebro del perro geriátrico", (Tesis Doctoral). Facultad de Veterinaria. Universidad de León. León, España, 2008.

[38] J. Gili, Introducción biofísica a la resonancia magnética. Barcelona, España: Ed Centre Diagnóstic Pedralbes, 1993.

[39] X.L. Jaramillo-Chaustre, A.E. Serantes-Gómez, \& J.J. Bustamante-Cano, "Caracterización de la intensidad de señal de la glándula mamaria mediante imagen por resonancia magnética en modelo animal canino", Iteckne, 13(2), pp. 137-145. Retrieved January 29, 2017, from http://www.scielo.org.co/scielo.php?script=sci arttext\&pid=S1692-17982016000200004\&Ing=en\& tIng=es. (2016)

[40] A.G, Dean, A.J. Dean, D. Coulombier, A.H. Burton, K.A. Brendel, D.C. Smith, R.C. Dicker, K.M. Sullivan, R.F. Fagan, Epi Info, Version 6,04: a word processing, database, and statistics program or epidemiology on microcomputer, 1994.

[41] A. Desgrez, J. Bittoun, I. Idy-Peretti, "Bases físicas de la IRM”. Cuadernos de IRM. Barcelona, España: Ed Mason, S.A., 1991.

[42] S. Kii, Y. Uzuka, Y. Taura, M. Nakaichi, A. Takeuchi, H. Inokuma, T. Onishi, "Magnetic resonance imaging of the lateral ventricles in beagle-type dogs". Veterinary Radiology \& Ultrasound, vol. 38 (6), pp. 430-433, 1997.

[43] B.E. Ratsch, S. Kneissl, C. Gabler, "Comparative evaluation of the ventricles in the Yorkshire terrier and the German shepherd dog using low-field MRI", Veterinary Radiology \& Ultrasound, vol. 42 (5), pp. 410-413, 2001.

[44] T. Vullo, E. Korenman, R.P. Manzo, D.G. Gómez, M.D.F. Deck, P.T. Cahill, "Diagnosis of cerebral ventriculomegaly in normal adult Beagles using quantitative MRI", Veterinary Radiology \& Ultrasound, vol.38 (4) pp. 277281, 1997.

[45] M-Y. Su; E. Head, W.M. Brooks, Z. Wang, B. Muggenburg, G.E. Adam, R. Sutherland, C.W. Cotman, 0 . Nalcioglu, "Magnetic resonance imaging of anatomic and vascular characteristics in a canine model of human aging", Neurobiology of Aging, vol. 19 (5), pp. 479-485, 1998.

[46] M-Y. Su, E. Head, B. Muggenburg, G.J-Y. Chiou, J. Wang, W.M. Brooks, R, Lee, C.W. Cotman, O. Nalcioglu, MRI measurement of changes in the aging canine brain, presented at the Symposium on Brain Aging and Related Behavioral Changes in Dogs, pp. 13-16, 2002.

[47] V. Ho, S. Allen, M. Hood, "Renal Masses: Quantitative Assessment of Enhancement with Dynamic MR Imaging", Radiology, vol. 224. pp. 695-700, 2002.

[48] D. Tapp, C.T. Siwak, F.Q. Gao, J.Y. Chiou, S.E. Black, E. Head, B.A. Muggenburg, C.W. Cotman, N.W. Milgram, L.M. Su, "Frontal lobe volume, function, and $\beta$-amyloid pathology in a canine model of aging", Journal of Neuroscience, vol. 24 (38), pp. 8205-8213, 2004.

[49] P.D. Tapp, K. Head, E. Head, N.W. Milgram, B.A Muggenburg, M-Y. Su, "Application of an automated voxelbased morphometry technique to assess regional gray and white matter brain atrophy in a canine model of aging", Neuroimage, vol. 29 pp. 234-244, 2006.

[50] R. Dennis, "An introduction to veterinary CT and MR scanning". Vet. Annual. 36, pp. 17-40, 1996.

[51] M. Snellman, Magnetic resonance imaging in canine spontaneous neurological disorders: an evaluation of equipment and methods, Academic dissertation. University of Helsinki, Finland. 2000, pp. 32-64.

[52] D. Thrall, "Principios físicos de la tomografía computarizado y de la resonancia magnética". Manual de Diagnóstico Radiológico Veterinario, $4^{\mathrm{a}}$. ed. Clifford R. Berry, Cap. 3, 2003.

[53] S.H. Heywang, T. Hilbertz, R. Beck, W.M. Bauer, W. Eiermann, W. Permanetter, "Gd-DTPA enhanced MR imaging of the breast in patients with postoperative scarring and silicon implants", J Comput Assist Tomo-

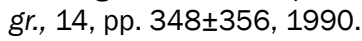

[54] T.H. Helbich, "Contrast-enhanced magnetic resonance imaging of the breast". European Journal of Radiology 34, pp. 208-219. Department of Radiology, University of Viena, 2000. 
[55] J.P. Stack, O.M. Redmond, M.B. Codd, P.A. Dervan, J.T. Ennis, "Breast disease: tissue characterization with Gd- DTPA enhancement profiles", Radiology, 174(2), pp. 491-4, 1990.

[56] G. Pons i Playà, "Planificación Pre-operatoria de Anastomosis Linfático-venosas mediante ICG-Linfografía y Linfo-Resonancia Magnética". (Tesis Doctoral). Universidad Autónoma de Barcelona, Barcelona, España, 2015.

[57] L.W. Nunes, M.D. Schnall, S.G. Orel, "Update of breast MR imaging architectural interpretation model". Radiology, 219 (2), pp. 484-94, 2001.
[58] S.G. Orel, M.D. Schnall, “MR imaging of the breast for the detection, diagnosis, and staging of breast cancer", Radiology, 220, pp. 13-30, 2001.

[59] T. Kinoshita, T. Fukutomi, K. Kubochi, "Magnetic Resonance Imaging of Benign phyllodes tumors of the breast", The Breast Journal, vol. 10, No. 3, pp. 232236, 2004.

[60] L.M. Mitsumori, E.S. McDonald, G.J. Wilson, P.C. Neligan, S. Minoshima, J.H. Maki, "MR lymphangiography: How i do it”, J Magn Reson Imaging, 42(6), pp. 1465-77. doi: 10.1002/jmri.24887. Epub 2015 Apr 23. 2015. 